\title{
A database of genetically determined neurological conditions for clinicians
}

\author{
MICHAEL BARAITSER, * KIYOTAKA TOMIWA, JOHN WILSON,* ROBIN WINTER† \\ From the Hospital for Sick Children, Great Ormond Street, London, ${ }^{*}$ and the Kennedy Galton Centre, \\ Northwick Park Hospital, $\uparrow$ Harrow, Middlesex, UK
}

SUMMARY Progress is reported in the establishment of a database of genetically determined neurological conditions. There are now 1,300 conditions and 3,400 references stored on the hard disk of a microcomputer. The database is searched, and a differential diagnosis obtained, by choosing a small number of signs and symptoms from a comprehensive list of cardinal features.

Many neurological conditions are chronic, progressive and to some degree genetically determined. If congenital brain anomalies are added to the list, there are over 1,000 known and possibly recognisable conditions which need to be diagnosed and in which counselling needs to be offered. This number is clearly too large for the average neurologist to retain in the memory, but a literature search is usually not possible until the condition has been named. Fortunately, the situation has now changed, because computer techniques are available to allow easy storage and retrieval of data for clinical use.

We present the progress that has been made in achieving a neurogenetic database which has been developed in conjunction with the London Dysmorphology Database. ${ }^{12}$

The aim of the database is to assist neurologists in making a diagnosis and is designed to augment their skills and not replace them. The end result is to provide a short-list of possible diagnoses, given an input of the relevant clinical features, or to provide all the important references for a known diagnosis. A comprehensive list of the clinical features for any genetically determined neurological condition can also be displayed.

Address for reprint requests: Dr M Baraitser, The Hospital for Sick Children, Great Ormond Street, London WCI 3JH

Received 22 April 1988

Accepted 26 August 1988

\section{Method}

Computer programs were written in Dbase III, a commercially available database management system. The programs can be run on any microcomputer with a hard disk installed with Dbase III. The database consists of three major files; a code file, a syndrome feature file, and a reference file.

\section{The Code file}

A full list of 1120 non-neurological clinical signs, covering every part of the body, had been previously drawn up and used in the London Dysmorphology Database. To this $\mathbf{3 8 0}$ neurological symptoms and signs were added. They include most clinical features, pathological and special investigations used by neurologists. Each sign or symptom is given a three level code. In the table the codes for extrapyramidal abnormalities are shown. The first level of the code is 32.Neurology. The second level of the code is 32.18 , Extrapyramidal. For the third level of the code, extrapyramidal signs are broken down into their various components. This allows a flexible approach to searching. For example, in a family some affected individuals may have dystonia whereas others may have athetosis. In this situation it might be better to search on 32.18 .00 , that is, something wrong with the extrapyramidal system, rather than on the third level codes for specific extrapyramidal signs.

Individual searches are quick and easy so that if one strategy is unrewarding, that is, the list of possible diagnoses is too long or too short, another strategy can be tested.

\section{Syndrome file}

Each syndrome is given an abreviated title (syndrome mnemonic) and a more descriptive full title. Up to 28 clinical 
Table 32.18.00 Extrapyramidal signs

\begin{tabular}{ll}
\hline 32.18 .01 & rigidity \\
32.18 .02 & chorea \\
32.18 .03 & athetosis \\
32.18 .04 & dystonia \\
32.18 .05 & tremor at rest \\
32.18 .06 & myoclonus \\
\hline
\end{tabular}

features can be entered for each syndrome using the three level codes. Conditions which look similar but seem clinically different, that is, they have different ages of onset or inheritance patterns are registered separately.

\section{Reference file}

Any number of references for each syndrome can be entered. Each record consists of the syndrome mnemonic, authors' names, date, journal name, volume, page number as well as a single line comment on the publication.

Entry of data

Previously, genetic syndromes from all the paediatric, orthopaedic, ophthalmological, genetic and most general journals had been examined and entered into the dysmorphology database. All syndromes with neuromuscular involvement were transferred to the neurogenetic database. Further syndromes and references were entered from the literature by "paging through" all the adult and paediatric neurology journals over the past 15 years. Each article of neurogenetic interest was entered as a reference if the syndrome described had already been registered or as a new syndrome if it had not, in which case all the clinical characters and the findings on examination and special investigations were entered. Experience has taught that a clinician is essential for data entry. For example, a report described a patient with progressive spasticity with brisk reflexes, ankle clonus and extensor plantar responses. Those entering the data must make a value judgement as there is no code for a Babinski sign, but there is little doubt that the patient in question has pyramidal tract involvement which can be entered as a specific code. In essence it is not possible or advisable to enter minute details of history, examination or investigations but to extract from each case the essentials. Each syndrome has as many references as is thought necessary to be useful for the neurologist. Very rare neurological syndromes are included even if they have only been described once. Most neurologists will have access to the literature and possible diagnoses can be considered by consulting the original report. At present 1300 neurogenetic conditions with 3400 references are stored in the database.

\section{Searching the Database}

Experience has shown that it is best not to enter detailed clinical features and expect an exact match. Clinical neurology is far too variable to match all symptoms precisely and individual clinicians use different terms to describe the same phenomenon. For instance a patient with an adult onset cerebellar ataxia might have a pigmentary retinopathy. There are separate codes for retinitis pigmentosa as in the simple $X$ linked, recessive or dominant types, as well as a code for atypical pigmentary retinopathy, and this might lead to confusion. It may be more productive to ask the computer for those conditions having a cerebellar ataxia and any retinal abnormality.
Example

Two sibs, an 11 year old boy and his elder sister, suffered from a neurodegenerative condition with sensorineural deafness optic atropy and spasticity starting in late infancy. A syndrome search using sensorineural deafness, 06.06.05, optic atrophy, 07.09.03, and spasticity 32.17.01 gave a list of only four conditions; using more generalised categories of deafness, pyramidal signs, and optic atrophy, nine conditions were suggested.

1. Adrenoleukodystrophy

2. Spongy degeneration (Canavan)

3. Friedreich's ataxia

4. Infantile neuroaxonal dystrophy (Seitelberger's disease)

5. Krabbe's disease

6. Multiple sulfatase deficiency

7. Optico-cochleo-dentate degeneration

8. Progressive pontobulbar palsy with deafness (VialettoVan Laere)

9. Progressive infantile poliodystrophy [Alpers].

On review the laboratory and pathological investigations suggested the diagnosis of optico-cocholeo-dentate degeneration. It was however useful to have a reasonable number of conditions as a differential diagnosis in order not to miss the true diagnosis.

Patients with a degenerative illness of the nervous system with many neurological problems but who had on special investigation calcification of the basal ganglia should be searched for on age of onset, 32.01.—, neurodegeneration, 32.04.03, basal ganglia calcification 32.31.02. The user will probably get a small but useful list which an experienced clinician will be able to scrutinise to exclude the majority before looking at the small number of remaining conditions to see whether they correspond.

\section{Conclusion}

There are to date 1300 neurological conditions in the database plus 3400 references. The purpose of the neurogenetic database is to give neurologists easy access to a vast amount of information on genetically determined neurological conditions including those that are extremely rare. It is essential that the database is always up-dated, edited, and new findings added. The authors have drawn on the experience with the London Dysmorphology Database to maintain the database and update it by constantly referring to new publications. The program is available to all neurologists who wish to use it.

We would like to acknowledge with thanks a generous grant from the Children' Nationwide Medical Research Fund.

\section{References}

1 Winter RM, Baraitser M, Douglas J. A computerised data base for the diagnosis of rare dysmorphic syndromes. J Med Genet 1984;21:121-3.

2 Tomiwa K, Baraitser M, Brett EM, Wilson J. The use of a computerised database for the diagnosis of a rare neurological syndrome. Neuropaediatrics 1987;18: 231-4. 